University of Nebraska - Lincoln

DigitalCommons@University of Nebraska - Lincoln

$9-24-2001$

\title{
Threshold Effects on Angular Distributions for Multiphoton Detachment by Intense Elliptically Polarized Light
}

\author{
Bogdan Borca \\ University of Nebraska - Lincoln
}

M.V. Frolov

Voronezh State University, Voronezh, Russia

N. L. Manakov

Voronezh State University, manakov@phys.vsu.ru

Anthony F. Starace

University of Nebraska-Lincoln, astarace1@unl.edu

Follow this and additional works at: https://digitalcommons.unl.edu/physicsstarace

Part of the Physics Commons

Borca, Bogdan; Frolov, M.V.; Manakov, N. L.; and Starace, Anthony F., "Threshold Effects on Angular Distributions for Multiphoton Detachment by Intense Elliptically Polarized Light" (2001). Anthony F. Starace Publications. 80.

https://digitalcommons.unl.edu/physicsstarace/80

This Article is brought to you for free and open access by the Research Papers in Physics and Astronomy at DigitalCommons@University of Nebraska - Lincoln. It has been accepted for inclusion in Anthony F. Starace Publications by an authorized administrator of DigitalCommons@University of Nebraska - Lincoln. 


\title{
Threshold Effects on Angular Distributions for Multiphoton Detachment by Intense Elliptically Polarized Light
}

\author{
Bogdan Borca, ${ }^{1, *}$ M. V. Frolov, ${ }^{2}$ N. L. Manakov, ${ }^{2}$ and Anthony F. Starace ${ }^{1}$ \\ ${ }^{1}$ Department of Physics and Astronomy, The University of Nebraska, Lincoln, Nebraska 68588-0111 \\ ${ }^{2}$ Department of Physics, Voronezh State University, 394693 Voronezh, Russia
}

(Received 7 May 2001; published 10 September 2001)

\begin{abstract}
Unusual threshold effects are found theoretically in the frequency and intensity dependence of angular distributions produced by an elliptically polarized laser as well as in the elliptic dichroism parameter, which measures the asymmetry of the angular distribution.
\end{abstract}

PACS numbers: $32.80 . \mathrm{Rm}, 32.80 . \mathrm{Gc}, 32.80 . \mathrm{Qk}$

The use of elliptically polarized laser light fields has added a new dimension to the study of multiphoton processes. Although the first theoretical analysis of the ionization of a bound electron by an elliptically polarized field was carried out in 1966 [1] (as a generalization of the well-known Keldysh results for linear polarization), it was the experimental observation in 1988 [2] of asymmetries in above threshold ionization (ATI) angular distributions (ADs) from rare gas atoms that sparked much subsequent work. The observed asymmetries cannot be described within the Keldysh approximation, but require explicit treatment of the binding potential [3-5]. Subsequently, the general form of ATI ADs was analyzed for detachment from both $s$ states [6] and closed $p$ subshells [7], their dependence on the laser ellipticity was both analyzed [8] and measured [9], and realistic numerical predictions for the $\mathrm{H}^{-}$ion were given [10]. Most recently, the necessary conditions for elliptic dichroism (ED) in 2-photon ionization have been analyzed [11], elliptically polarized light has been used to perform complete experimental 2-photon ionization measurements [12], and the role of rescattering in ATI with elliptically polarized light has been studied $[13,14]$. Note that these studies of ATI ADs have generally emphasized the ellipticity dependence for particular laser frequencies and intensities.

In this Letter we present accurate numerical results on the global frequency and intensity dependence of the multiphoton detachment ADs produced by elliptically polarized light and of the corresponding ED, both of which are found to show unusual features in the vicinity of multiphoton detachment thresholds. Wigner-cusp features in above threshold detachment (ATD) and in high-order harmonic generation spectra were predicted some time ago $[15,16]$. For ADs and ED produced by elliptically polarized light we find other features that appear in both their frequency and their intensity dependences. Specifically, (i) as the laser intensity increases and nears the closing of the lowest-order detachment channel, the probability for electron ejection along the major polarization axis tends to zero; i.e., the ejected electrons have a significant velocity component in the plane perpendicular to the major polarization axis. (ii) ED parameters for odd-photon ATD peaks are found to be zero (nonzero) for frequencies below (above) the threshold of the next lower evenphoton ATD channel; also, the ED parameters alternate their signs in the vicinity of the opening of each new ATD channel. (iii) As a function of intensity, the ED parameter exhibits a decreasing trend from positive values at low intensities, passing through zero to negative values with increasing laser intensity and exhibiting a thresholdrelated staircase-type behavior. Our results were obtained using a quasistationary quasienergy state (QQES) approach [17] for the detachment of an electron bound in a three-dimensional, zero-range potential. This approach permits essentially exact predictions for laser intensities extending from the perturbative to the nonperturbative intensity regime and for frequencies extending from the tunneling to the multiphoton regimes. In particular, this approach treats accurately the effects due to the binding potential, which are necessary to obtain the proper symmetries for the ADs produced by elliptically polarized light. Note that our approach has the flexibility to reduce either to the Keldysh approximation, if binding potential effects on the escaping electron are ignored, or to the perturbative results, if an expansion in the laser intensity is performed.

We consider an electron in a three-dimensional, zerorange, $\delta$-model potential interacting with an elliptically polarized field treated in the dipole approximation:

$$
\mathbf{F}(t)=F \operatorname{Re}\left\{\mathbf{e} e^{-i \omega t}\right\}, \quad \mathbf{e}=\frac{\hat{\boldsymbol{\epsilon}}+i \eta[\hat{k} \times \hat{\epsilon}]}{\sqrt{1+\eta^{2}}},
$$

where $\hat{k}$ is the laser propagation direction, $\hat{\boldsymbol{\epsilon}}$ is the direction of the major polarization axis, and $\eta$ is the ellipticity. For this model, the QQES approach leads to the following eigenvalue problem for the complex quasienergy $\epsilon=E_{0}+\Delta-i \Gamma / 2$, where $\Delta$ is the Stark shift and $\Gamma$ is the total decay rate of the bound level [18]:

$$
\left(\sqrt{2 n \omega+\gamma^{2}-\epsilon}-1\right) \phi_{n}=\sum_{n^{\prime}=-\infty}^{\infty} M_{n, n^{\prime}}(\epsilon) \phi_{n^{\prime}} .
$$

We measure energies (and $\hbar \omega$ ) in units of the unperturbed binding energy $\left|E_{0}\right|$ and the peak laser field strength $F$ in units of $F_{0}=\sqrt{2 m\left|E_{0}\right|^{3}} /|e| \hbar$. We also use the parameter $\gamma=F /(\sqrt{2} \omega)=1 /\left(\sqrt{2} \gamma_{K}\right)$, where $\gamma_{K}$ is the 
well-known Keldysh parameter. The $\phi_{n}$ in Eq. (1) are Fourier coefficients of the periodic in time QQES wave function, $\Phi_{\epsilon}(\mathbf{r}, t)$, at the origin $(|\mathbf{r}| \rightarrow 0)$. The matrix elements $M_{n, n^{\prime}}(\epsilon)$ involve one-dimensional integrals of Bessel functions, which can be evaluated using either a series expansion in $\gamma^{2} / \omega$ [18] or an analytic continuation procedure [19] (for high intensities, when the series expansion is slowly convergent), which requires a more substantial numerical effort. Using the asymptotic form at $|\mathbf{r}| \rightarrow \infty$ for the properly-normalized function $\Phi_{\epsilon}(\mathbf{r}, t)$ (cf. Ref. [20]), the differential $n$-photon detachment rate in the direction $\mathbf{n}$ (defined by spherical angles $\theta$ and $\varphi$ in a frame with the $Z$ axis along $\hat{k}$ and the $X$ axis along $\hat{\epsilon}$ ) is

$$
\begin{gathered}
\frac{d W^{(n)}}{d \Omega}=2\left|\sqrt{k_{n}} \mathcal{A}_{n}\right|^{2} \\
\mathcal{A}_{n}=(-1)^{n} \sum_{p=-[n / 2]}^{\infty} \phi_{p} J_{2 p+n}\left(\frac{2 F k_{n}}{\omega^{2}}|\mathbf{n} \cdot \mathbf{e}|\right)\left(i \frac{\mathbf{n} \cdot \mathbf{e}}{|\mathbf{n} \cdot \mathbf{e}|}\right)^{2 p} \\
+\sum_{p=[n / 2]+1}^{\infty} \phi_{-p} J_{2 p-n}\left(\frac{2 F k_{n}}{\omega^{2}}|\mathbf{n} \cdot \mathbf{e}|\right)\left(i \frac{\mathbf{n} \cdot \mathbf{e}^{*}}{|\mathbf{n} \cdot \mathbf{e}|}\right)^{2 p}
\end{gathered}
$$

where $k_{n}=\sqrt{\epsilon+n \omega-\gamma^{2}}$ is the complex "photoelectron momentum," $n \geq\left[|\operatorname{Re} \epsilon|+\gamma^{2}\right] / \omega$, and $[x]$ is the largest integer less than $x$.

The symmetries of ADs and the existence of ED (defined as a difference in $d W^{(n)} / d \Omega$ for $\eta \rightarrow-\eta$ or, equivalently, for $\mathbf{e} \rightarrow \mathbf{e}^{*}$ ) are established from the explicit dependence of the amplitude $\mathcal{A}_{n}$ on the polarization-angular phase factor $[(\mathbf{n} \cdot \mathbf{e}) /|\mathbf{n} \cdot \mathbf{e}|]^{2} . \mathcal{A}_{n}$ is given by the exact result (3), where the coefficients $\phi_{ \pm p}$ depend only on the degree of linear polarization $l=\left(1-\eta^{2}\right) /\left(1+\eta^{2}\right)$; i.e., they are independent of the sign of $\eta$. The ED terms in the AD (2) are those proportional to odd powers of

$$
\operatorname{Im}\left\{(\mathbf{n} \cdot \mathbf{e})^{2}\right\}=\xi(\mathbf{n} \cdot \hat{\boldsymbol{\epsilon}})(\mathbf{n} \cdot[\hat{k} \times \hat{\boldsymbol{\epsilon}}]),
$$

where $\xi=2 \eta /\left(1+\eta^{2}\right)$ is the circular polarization degree. This factor (and hence ED) vanishes for the case of circular polarization, $\xi= \pm 1$, since only the coefficient $\phi_{n}$ with $n=0$ in Eqs. (1) and (3) is nonzero for this case [18]. In general, inversion symmetry of the AD exists due to parity conservation, i.e., the $\mathrm{AD}$ is invariant to $\mathbf{n} \rightarrow-\mathbf{n}$. Also, the $\mathrm{AD}$ is invariant to a rotation by an angle $\pi$ around the direction of $\hat{k}$, since this equals a simultaneous rotation by an angle $\pi$ around the directions of $\hat{\boldsymbol{\epsilon}}$ and $[\hat{k} \times \hat{\boldsymbol{\epsilon}}]$. Neglecting the ED terms, ADs exhibit an additional symmetry with respect to separate rotations by an angle $\pi$ around either $\hat{\epsilon}$ or $[\hat{k} \times \hat{\epsilon}]$. The ED terms destroy this symmetry and cause the well-known asymmetry of ADs originating from the ED effect. Thus the asymmetry of ADs illustrates an interesting physical phenomenon specific to an elliptically polarized field: the light-helicity dependence of multiphoton cross sections, which vanishes for the case of pure circular polarization. We note also the general symmetry of ADs with respect to the substitution $\xi, \hat{k} \rightarrow-\xi,-\hat{k}$, i.e., the equivalence of ADs in two experiments with opposite helicities and directions of a laser beam. Obviously, if one neglects the coefficients $\phi_{n}$ (as is done to reduce the exact results for a zero-range potential to the Keldysh approximation [18,21], which is equivalent to neglecting the effect of the binding potential on the escaping electron in both intermediate and final states [22]), the $\mathrm{ED}$, and thus the asymmetry of the $\mathrm{AD}$, vanishes. All of these symmetries and the ED effect are clearly seen in Fig. 1, which shows the three-dimensional ADs for 3photon detachment at $\eta= \pm 0.4$ for $\omega=0.4$ (in which case there is no ED, see below) and 0.8.

To analyze the dependence of the ED on frequency and intensity, we introduce the ED parameter, $\delta^{(n)}$ :

$$
\delta^{(n)}=\frac{d W^{(n)}(\eta) / d \Omega-d W^{(n)}(-\eta) / d \Omega}{d W^{(n)}(\eta) / d \Omega+d W^{(n)}(-\eta) / d \Omega},
$$

where the differential rates are measured in the direction of the geometrical maximum of the effect [cf. Eq. (4)]: $\theta=\pi / 2$ (i.e., in the polarization plane) and $\varphi=\pi / 4$ (i.e., halfway between the major and minor axes of the polarization ellipse). In Fig. 2 we plot $\delta^{(n)}$ (for $n=2,3,4,5$ ) as a function of $\omega$ at low intensity, i.e, in the perturbative regime, where the AD does not depend on the intensity. The $n$-photon rate exists only for $\omega>1 / n$ (the $n$-photon threshold). The most remarkable feature is that both the 3- and 5-photon processes do not present any asymmetry over the ranges $1 / 3<\omega<1 / 2$ and $1 / 5<\omega<1 / 4$ respectively (cf. Fig. 2 for $n=3,5$ ). Similar observations can be made for any odd $n$. We explain this effect using the lowest order perturbation theory (LOPT) limits of Eqs. (1) and (3). As an example, in the LOPT we obtain the following analytic expression for $\mathcal{A}_{3}$ :

$$
\mathcal{A}_{3}=\frac{k_{3} F}{\omega^{2}}(\mathbf{n} \cdot \mathbf{e})\left[\phi_{-1}-\phi_{0} \frac{\left(k_{3} F\right)^{2}}{6 \omega^{4}}(\mathbf{n} \cdot \mathbf{e})^{2}\right] .
$$

Since, in the LOPT, $\phi_{0}=1 / \sqrt{2 \pi}$ and $k_{n}=\sqrt{n \omega-1}$, the key to understanding the peculiar behavior of the asymmetry lies in the $\omega$ dependence of $\phi_{-1}$ :
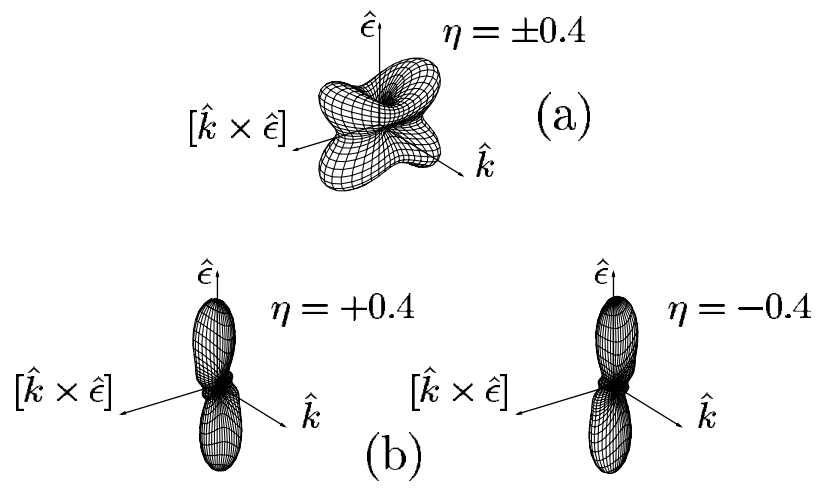

FIG. 1. 3-photon detachment $\mathrm{AD}$ at (a) $\omega=0.4$ and (b) 0.8 and low intensity for right and left elliptically polarized light. 


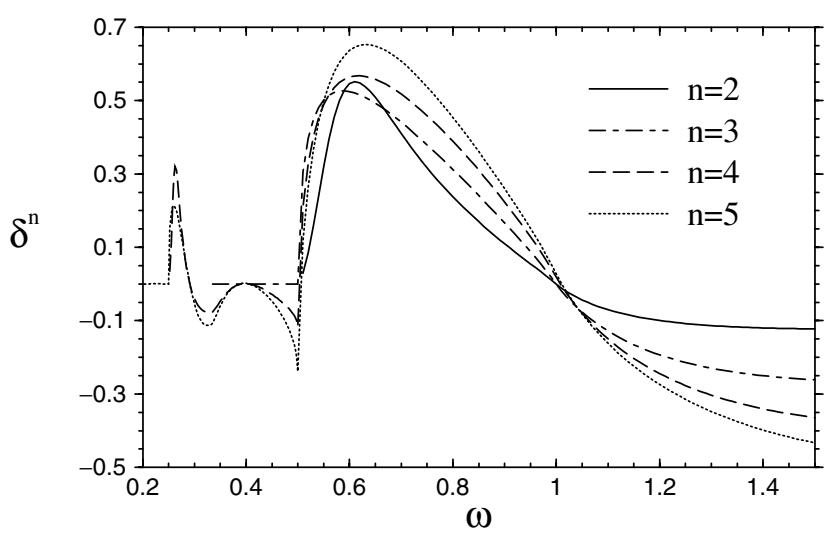

FIG. 2. The ED parameter $\delta^{(n)}$ vs frequency, for the low intensity (perturbative) regime. Ellipticity is $\eta=0.4$.

$$
\phi_{-1}=\frac{l F^{2}}{\sqrt{2 \pi} 6 \omega^{4}} \frac{i(2 \omega-1)^{3 / 2}-2 i(\omega-1)^{3 / 2}+1}{1+i \sqrt{2 \omega-1}}
$$

$\operatorname{Im}\left\{\phi_{-1}\right\}$ is zero for $\omega$ below $1 / 2$ and nonzero above the 2 -photon threshold. Subsequently, $\operatorname{Im}\left\{\phi_{-1}\right\}$ changes its sign at the 1-photon threshold, which is reflected in a sign change of $\delta^{(3)}$ at $\omega=1$ (cf. Fig. 2). The LOPT expression for $\mathcal{A}_{2}$, on the other hand, involves the same coefficients, $\phi_{0}$ and $\phi_{-1}$, and, therefore, starting from the threshold for this process $(\omega=1 / 2), \operatorname{Im}\left\{\phi_{-1}\right\}$ is nonzero and so is the parameter $\delta^{(2)}$. In general, for the $2 p$ and $2 p+1$ processes for $p \geq 1$, the key role is played by $\operatorname{Im}\left\{\phi_{-p}\right\}$, which is zero below $\omega=1 /(2 p)$ and changes sign in the vicinity of each higher threshold up to $\omega=1$.

We consider now how ADs are modified as the laser intensity increases from the perturbative to the nonperturbative regime for the cases in which the detached electrons absorb $n$ elliptically polarized photons with $\eta=0.4$. For $\omega=0.8$, Fig. 3 displays the 2- and 3-photon ADs from low laser intensities to the intensities corresponding to the closures of those particular channels. (The closing of channels involving lower numbers of photons as the intensity increases is well known. It is due mainly to the increase of the ponderomotive threshold shift, $U_{p} \equiv \gamma^{2}$, which cannot be compensated by the much smaller Stark shift, $\Delta$, of the bound level.) At low intensity, the ADs are strongly peaked along the major axis of the polarization ellipse (i.e., the vertical axis in Fig. 3). As the intensity increases, the probability of electrons being ejected along this axis decreases and more electrons are ejected perpendicular $(n=2)$ or at an angle $\varphi \approx \pi / 4(n=3)$. For each case, at some intensity before the channel closing, no electrons are ejected along the major polarization axis. This effect is caused by significant interference of terms with different $p$ in Eq. (3) for the detachment amplitude in the nonperturbative regime and occurs (for slightly different values $F$ ) for an arbitrary polarization, except purely circular, including linear polarization. For intensities still
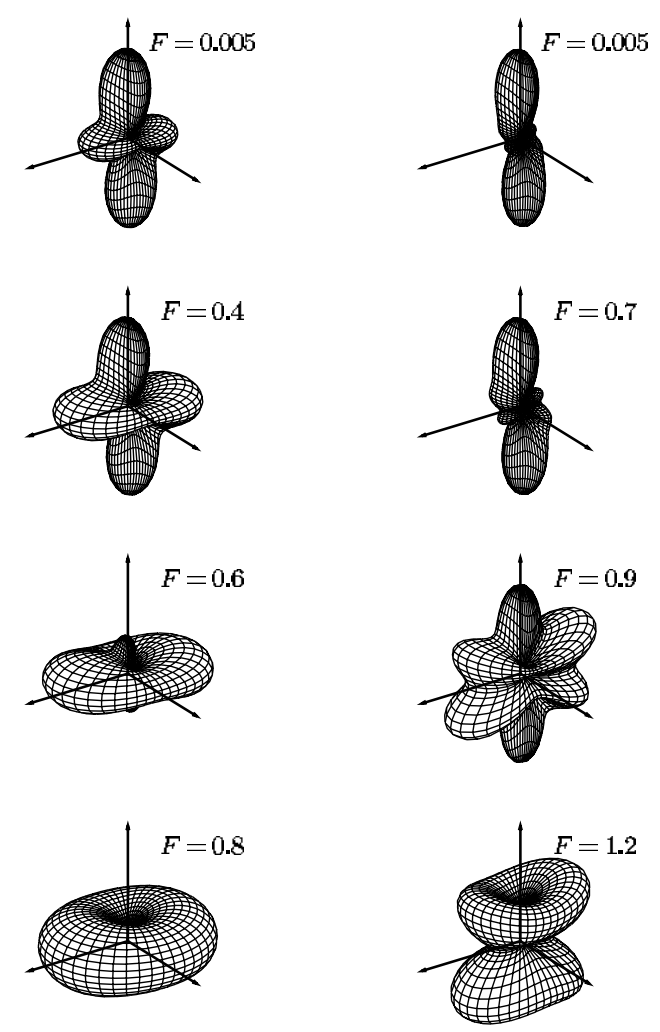

FIG. 3. 2-photon (left) and 3-photon (right) detachment ADs at $\omega=0.8$ and $\eta=0.4$ and several values of the peak field strength, as indicated in the figure. The directions of $\hat{\epsilon}$ and $\hat{k}$ are the same as Fig. 1. The different figures have been scaled so that they have the same size.

closer to threshold, the AD's revert to those expected for the lowest photoelectron angular momentum allowed by parity selection rules (e.g., $L=0$ or 1 ), in accordance with Wigner's threshold law, i.e., the AD is isotropic (for even $n$ ) or $\cos ^{2} \theta$ dependent (for odd $n$ ). Similar situations were found by us in calculations of other $n$-photon detachment rates for $n>3$.

The asymmetry of the ADs is seen in Fig. 3 to be strongly connected with the electrons ejected along the main polarization axis. As $F$ increases and electrons are ejected perpendicularly or obliquely to $\hat{\epsilon}$, the ED decreases and at the limit of the channel closing, when Wigner's threshold law applies, the asymmetry tends to 0 . We plot in Fig. 4 the ED parameter $\delta$ corresponding to the total detachment yield $d W(\eta) / d \Omega=\sum_{n \geq n_{\min }} d W^{(n)}(\eta) / d \Omega$. At lower intensities, for each of the frequencies considered, the dominant contribution is given by the channel involving the minimum number of photons required for detachment. The deep minima correspond to the closing of different channels. As the intensity increases, above threshold detachment plays a more important role: the process involving the minimum number of photons for detachment (considering also $U_{p}$ ) is of similar magnitude to those involving more photons. Even though the higher intensity can significantly change the magnitude of the asymmetry, 


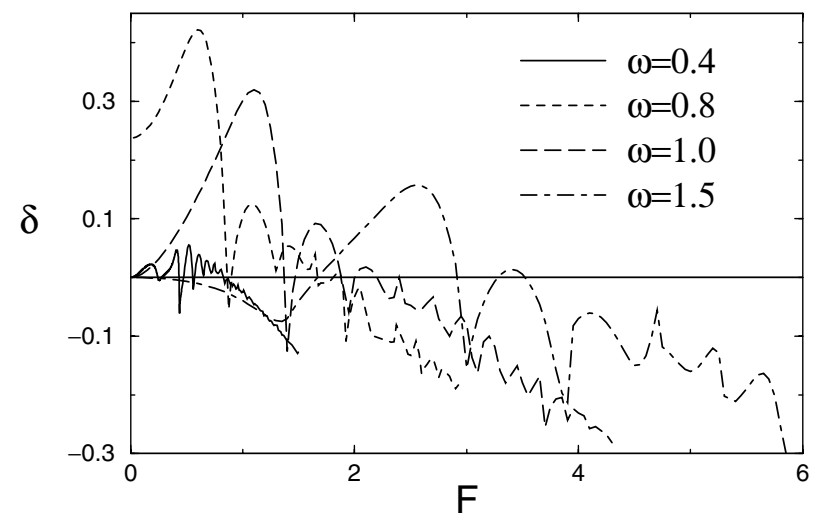

FIG. 4. Total ED parameter $\delta=[d W(\eta) / d \Omega-d W(-\eta) /$ $d \Omega] /[d W(\eta) / d \Omega+d W(-\eta) / d \Omega]$ as a function of field amplitude for four different frequencies. Ellipticity is $\eta=0.4$.

the scale on which these changes appear is strongly related to the magnitude of the asymmetry in the perturbative domain of intensities. For example, at $\omega=0.4$, the asymmetry is exactly 0 at low intensity and does not exceed 0.1 at high intensities. In contrast, for $\omega=0.8$ each $\delta^{(n)}(n \geq 2)$ has a considerable magnitude at low intensities and consequently so does the total $\delta$, including also when one increases the intensity. One sees in Fig. 4 that for all frequencies, as $F$ increases, there is a general decreasing trend of the asymmetry. The decrease continues below the value 0 so that the absolute value of the asymmetry increases at higher $F$. This trend is especially interesting at the low frequency, $\omega=0.4$, where it appears that an increase of $F$ will cause a significant asymmetry in spite of the small value of $\delta$ at small and medium values of $F$. However, for any $F$, as $\omega$ decreases the asymmetries in the ADs tend to decrease and vanish in the tunneling limit, $\omega \ll 1$.

In conclusion, we have presented novel threshold effects on the ADs of electrons detached from negative ions by an elliptically polarized field. As the laser intensity increases we find a decrease in the number of electrons ejected along the major laser polarization axis, becoming zero at an intensity close to the channel closing. This effect has an interference origin and cannot be explained in terms of the Wigner threshold law. We have analyzed the global frequency and intensity dependence of the ED, which measures the asymmetry of the ADs. The ED effect originates from the interference between real and imaginary parts of the detachment amplitude $\mathcal{A}_{n}$. Its correct description in the nonperturbative regime requires an exact account of both the imaginary part of the "momentum" $k_{n}$ in Eq. (3) and of the binding potential effects on the escaping electron. Regarding the applicability of our findings for actual atomic systems, we note that our approach agrees quite well with multielectron predictions for $\mathrm{H}^{-}$[23]; we expect the present results to apply to any system involving a short-range potential.

This work was supported by NSF Grant No. PHY0070980, by RFBR Grant No. 00-02-17843, and by Grant No. E00-3.2-515 of the Russian Ministry of Education.

*On leave from the Institute for Space Sciences, BucharestMagurele 76900, Romania.

[1] A. M. Perelomov et al., Zh. Eksp. Teor. Fiz. 51, 309 (1966) [Sov. Phys. JETP 24, 207 (1967)].

[2] M. Bashkansky et al., Phys. Rev. Lett. 60, 2458 (1988).

[3] S. Basile et al., Phys. Rev. Lett. 61, 2435 (1988); F. Trombetta et al., J. Phys. B 21, L539 (1988).

[4] P. Krstić and M.H. Mittleman, Phys. Rev. A 44, 5938 (1991).

[5] A. Jaroń et al., Opt. Commun. 163, 115 (1999).

[6] P. Lambropoulos and X. Tang, Phys. Rev. Lett. 61, 2506 (1988); H. G. Muller et al., ibid. 61, 2507 (1988).

[7] F. Dulieu et al., J. Phys. B 28, 3845 (1995); 28, 3861 (1995).

[8] S. P. Goreslavskii and S. V. Popruzhenko, Zh. Eksp. Teor. Fiz. 110, 1200 (1996) [Sov. Phys. JETP 83, 663 (1996)].

[9] G. G. Paulus et al., Phys. Rev. Lett. 80, 484 (1998).

[10] L. A. A. Nikolopoulos and P. Lambropoulos, Phys. Rev. A 56, 3106 (1997).

[11] N. L. Manakov et al., J. Phys. B 32, 3747 (1999).

[12] Z.-M. Wang and D. S. Elliott, Phys. Rev. Lett. 84, 3795 (2000); Phys. Rev. A 62, 053404 (2000).

[13] R. Kopold et al., Phys. Rev. Lett. 84, 3831 (2000).

[14] G. G. Paulus et al., Phys. Rev. Lett. 84, 3791 (2000).

[15] F. H. M. Faisal and P. Scanzano, Phys. Rev. Lett. 68, 2909 (1992).

[16] W. Becker et al., Phys. Rev. A 46, R5334 (1992).

[17] N. L. Manakov et al., Phys. Rep. 141, 319 (1996).

[18] N. L. Manakov and A. G. Fainshtein, Zh. Eksp. Teor. Fiz. 79, 751 (1980) [Sov. Phys. JETP 52, 382 (1980)].

[19] N. L. Manakov et al., Pis'ma Zh. Eksp. Teor. Fiz. 72, 426 (2000) [JETP Lett. 72, 294 (2000)].

[20] N. L. Manakov et al., J. Phys. B 33, R141 (2000).

[21] W. Becker et al., Phys. Rev. A 42, 4416 (1990).

[22] S. Geltman, Phys. Rev. A 42, 6958 (1990).

[23] M. V. Frolov et al., Phys. Rev. A 64, 023417 (2001). 